\title{
Instruments of granular matrix mechanics for solving design-level tasks on shear strength resistance of loosely-coupled material in cast stone pavement
}

\author{
Andrey Moshenzhal ${ }^{1}$, Svetlana Zhdanova ${ }^{2}$, and Aleksey Piotrovich ${ }^{2, *}$ \\ ${ }^{1}$ LLC "Stroyimpuls", Bldg. 47, Wing 2, Stachek Ave., St. Petersburg, 198097, Russia \\ ${ }^{2}$ Far Eastern State Transport University, 47 Seryshev St., Khabarovsk, 680021, Russia
}

\begin{abstract}
The paper covers the method of distribution analysis for normal and shear stresses in a multilayer cast stone pavement design. The method is used to test the shear strength requirements. Some conclusions about the expediency of considering the granular matrix discrete structure applied in transport construction are made.
\end{abstract}

\section{Introduction}

Improving the reliability of pavement and forecasting the service life of coatings with the aim of ensuring the safety of the roads are of primary importance for a designer. It is obvious that forecasting the service life of coatings, as well as ensuring their safety for the period of operation are impossible without taking into account the characteristics of the structural layers and their operation under the influence of the load. One of the criteria for the distribution of normal and shear stresses transmitted from layer to layer in depth is testing the ensurance of the conditions of shear resistance in soils. This is true for prefabricated structures made of artificial paving stones, for example, which have successfully proven themselves as a technological and reliable solution. The calculation methods should contain appropriate criteria for evaluating the effectiveness of the decisions made.

One of such criteria is checking the conditions of shear resistance in the soil. It is obvious that in order to provide it the designer needs to have a picture of the distribution of normal and tangential stresses transmitted from layer to layer in depth. The latter depends on the properties of structural layers and features of the transfer of pressure on the coating. This is especially important for modular designs, for example, made of artificial paving stone, that has successfully established itself as a technologically advanced and reliable solution.

The article touches upon the acute problem of the computational and theoretical substantiation of the model of shear resistance in weakly-cohesive soils of coatings made from artificial paving stones. Checking such a condition is necessary but the mechanism of transfer of pressure through the pavers on the loose media, as well as the mechanism for taking into account the nature of the distribution of normal and shear stresses in the calculations is studied very little. Providing a comprehensive forecasting of processes

\footnotetext{
*Corresponding author: piotrovich@ rambler.ru
} 
occurring in a discrete environment under the influence of surface load will allow us to take into account the peculiarities of the structures of weakly-cohesive soils and other structures for using them in transport construction.

\section{Results}

Artificial stone paving has proved its value as a material providing high technological and operational characteristics in comparison with other types of coatings [1]. The calculation of such structures is performed in accordance with the requirements $[2,3,4]$ for permissible elastic deflection and shear resistance.

To date, a promising direction in the study of stress distribution in granular layers of pavement structures coated with artificial paving stones is the mechanics of granular media [5]. The paper [6] points out the fact of the inclusion in the joint operation of several artificial stones outside the bounds of the applied load due to the friction in the joints between the stones. It is obvious that with different types of the filler the friction in each case and as a consequence the number of stones included in the operation will be different. In this case it is necessary to take into account the geometric parameters of pavers, the nature and location of the application of the surface load, as well as the features of the structures of the underlying layers of the soil.

The task of transfer and distribution of pressure through a similar coating structure can be divided into two parts. The first part is the determination of the values of the transmitted pressure from the die or wheel on the paving stones, the second one is the identification of the nature of the distribution of pressure from the stones on the underlying layers of the coating structure necessary for checking the conditions of shear resistance in weaklycohesive soils. In the papers $[7,8]$ we presented the solution for a flat setting that has obviously certain assumptions as it doesn't consider the spatial character of the transferred pressures from paving stones involved in this process. In the continued development of this topic, the present paper proposes a solution to this problem for a spatial setting.

For solving the problem in the spatial setting as well as in the flat setting one of the input parameters is determining the location of the load application, i.e. determining the number of paving stones involved in the transfer of pressure on the underlying layers. Here, the geometrical sizes of stones, and also the order of their arrangement ("drawing") on the prepared basis or constructive layers will be essential. As an example, let's consider the case of pressure transfer from uniformly distributed circle load on the paving stones (radius 0.17 $\mathrm{m}$ ) having the following geometric characteristics: width- $0.10 \mathrm{~m}$, length- $0.20 \mathrm{~m}$, thickness$0.07 \mathrm{~m}$ (Figures 1).

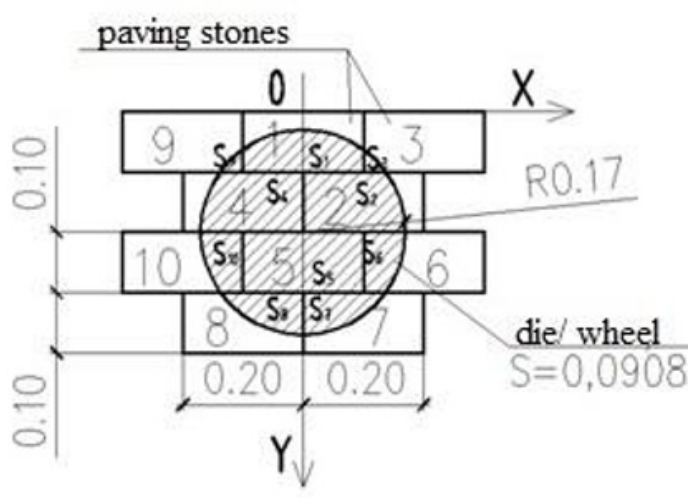

Fig. 1. The scheme for determining the number of stones involved. 
To solve this problem, we will introduce some assumptions:

- the pressure under the involved paving stones varies and depends on the area of the die support for each stone;

- the decision below does not take into account the friction forces between adjacent stones;

- when transferring the pressure on the stones, they do not cause torques.

To satisfy the first assumption, we will introduce the concept of the proportion of pressure $q i$ on a particular stone from the surface load.

This value is probabilistic and is defined for each stone as the ratio of the area of application of the surface load on the cobblestones $S i$ to the die area $S$ (1).

$$
q_{i}=\frac{s_{i}}{S}
$$

Here it should be noted that the sum of the values $q_{i}$ should be equal to one.

Using the method of sealing seams between stones is an important aspect both from the technical and economic points of view. It is obvious that when using the granite chips for this purpose between adjacent stones, for example, there emerge friction forces leading to an increase in the number of artificial stones involved in the transfer to the underlying structural layers of surface pressure. Thus, the stiffer the links between the stones, the larger the area of pressure distribution and the lower the values of normal and shear stresses in the soil. The satisfaction of the second assumption, namely the exclusion of friction forces, allows to achieve a certain reserve in the calculations, thereby providing greater reliability of decisions.

The coating of artificial paving stones is a system of non-dispersed blocks, i.e. the load is not distributed horizontally to adjacent stones due to the lack of friction forces between them. Thus, each individual stone is a small rectangular die with some special stress distribution beneath it. In this paper, we will present the solutions for the uniformly distributed load over a rectangle.

In the mechanics of granular media there are solutions (2) - (7) for determining the values of normal and shear stresses for a multilayer structure,

$$
\begin{gathered}
\sigma_{z}=\frac{p}{4}\left(\Phi\left(\frac{x+b}{(h+z) \sqrt{2 v_{i}}}\right)-\Phi\left(\frac{x-b}{(h+z) \sqrt{2 v_{i}}}\right)\right) \times\left(\Phi\left(\frac{y+a}{(h+z) \sqrt{2 v_{i}}}\right)-\Phi\left(\frac{y-a}{(h+z) \sqrt{2 v_{i}}}\right)\right), \\
h_{e}=h_{1} \sqrt{\frac{v_{1}}{v_{i}}}+h_{2} \sqrt{\frac{v_{2}}{v_{i}}}+\cdots+h_{i-1} \sqrt{\frac{v_{i-1}}{v_{i}}} \\
\tau_{x z}=-v z \frac{\partial \sigma_{z}}{\partial x} \\
\tau_{y z}=-v z \frac{\partial \sigma_{z}}{\partial y} \\
\sigma_{x}=v \sigma_{z}+v^{2} z^{2} \frac{\partial^{2} \sigma_{z}}{\partial x^{2}} \\
\sigma_{y}=v \sigma_{z}+v^{2} z^{2} \frac{\partial^{2} \sigma_{z}}{\partial y^{2}}
\end{gathered}
$$

where $p$ is the intensity of the applied pressure, $\mathrm{kN} / \mathrm{m}^{2}$ is the coefficient of distribution of the soil layer or base; $b, a$ - half the width of the load band along the $X$ axis and $Y$ axis 
respectively, $m ; X, Z, Y$ - coordinates of the test point in the XOZ and YOZ, $m ; \sigma_{z}$-vertical stress, $k N / \mathrm{m}^{2} ; \sigma_{x} \sigma_{y}$ - horizontal stress; $\mathrm{kN} / \mathrm{m}^{2} ; \tau_{x z} \tau_{y z}$ - shear stress, $\mathrm{kN} / \mathrm{m}^{2} ; h$ - thickness of the structural layer, $m$; $\Phi$ - error integral; $v$ - elastic modulus; $h_{e}$ - equivalent thickness of layer, $m$.

These equations allow to take into account the pressure transmitted by a single paving stone, but do not show the distribution of values $\sigma_{z}, \sigma_{x}$ И $\tau_{x z}$ from several surface loads, i.e. from the inclusion of multiple paving stones in the load transfer. In the mechanics of granular media this issue has not been previously considered. To solve this problem we will propose using the following equation (8):

$$
\sigma_{z}=\frac{p}{4} \sum_{i=1}^{n}\left(\Phi\left(\frac{x+\xi_{i}+l}{(h+z) \sqrt{2 v_{i}}}\right)-\Phi\left(\frac{x+\xi_{i}-l}{(h+z) \sqrt{2 v_{i}}}\right)\right) \times\left(\Phi\left(\frac{y+\chi_{i}+c}{(h+z) \sqrt{2 v_{i}}}\right)-\Phi\left(\frac{y+\chi_{i}-c}{(h+z) \sqrt{2 v_{i}}}\right)\right)
$$

with, $\xi_{\mathrm{i}}= \pm \mathrm{a}_{\mathrm{i}} ; \chi_{\mathrm{i}}= \pm \mathrm{f}_{\mathrm{i}}$, where 1 is half the length of paving stone, $\mathrm{m} ; \mathrm{c}$ - the half width of the paving stone, $\mathrm{m} ; \mathrm{a}_{\mathrm{i}}, \mathrm{f}_{\mathrm{i}}$ - the position of the center of the $\mathrm{i}$-th stone of the paving with respect to zero for the $X$ and $Y$ axes, respectively, $m ; n$ - number of involved paving stones in the process of transmission of pressure.

As it was noted earlier, in order to ensure a high reliability of coatings it is necessary to provide a condition of shear resistance in the structural layers or the basis made of weaklycohesive soils at the most critical point, which is characterized by the highest values of vertical stresses. In the spatial setting such a problem is complicated, because it is necessary to take into account the nature of the distribution of stresses from each element of the coating.

Using the above presented assumptions as well as the calculation scheme (Figure 1) let's determine the position of the critical point in terms of vertical stresses.

a)

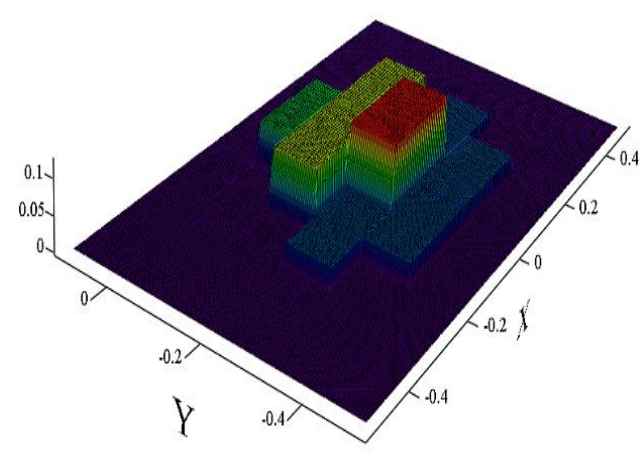

b)

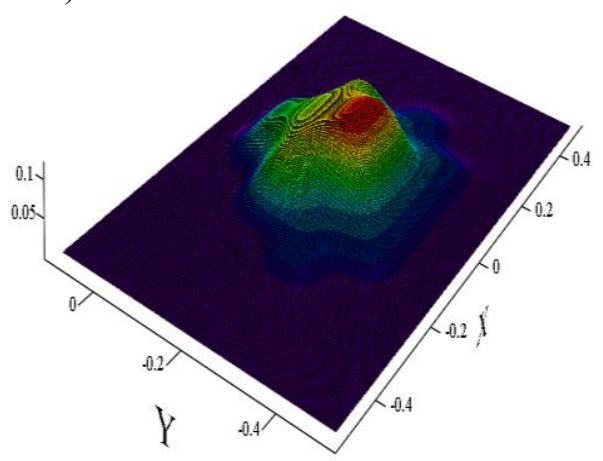

Fig. 2. The nature of the distribution of vertical stresses at different depths: a) $\mathrm{z}_{1}=0.01 \mathrm{~m}$; b) $\mathrm{z}_{2}=0.05$ $\mathrm{m}$.

For this purpose on the basis of the calculation scheme (Fig. 1) and equation (8) there was made a system of equations for 10 positions of the center of the $\mathrm{i}$-th paving stone with respect to zero on the $X$ and $Y$ axes (respectively $-a_{i}$ and $f_{i}, i=1,10$ ).

The calculations did not take into account the weight of the soil, and the value of the coefficient of the distribution capacity of the soil was equal to 0.350 .

Figure 2 shows the results of the calculated vertical stresses at different depths from the bottom of the paving stone.

According to the calculation scheme shown in Figure 1 the highest stresses occur under stone number 5 which is consistent with the results obtained (Figure 2.); 
The presented system of equations allows to determine the stress values at any point of the soil mass taking into account the unevenness of the pressure distribution over the paving stones.

Having a picture of the distribution of vertical stresses in the soil it will not be difficult to calculate the missing values of stresses using the formula (4)-(7), and check the conditions of shear resistance at the most critical point of the bulk soil or structural layer.

From the classical mechanics of soils it is known that the shear stability condition must be provided at all points of the soil mass and it is expressed by the inequation, in which the value of the active shear stresses must not exceed the permissible shear stress. According to [9] this condition can be represented with the following inequation (9):

$$
\begin{gathered}
\left(\sigma_{1}-\sigma_{2}\right) \sin 2\left(45 \pm \frac{\theta_{\max }}{2}\right)<\left[\left(\sigma_{1}+\sigma_{2}\right)+2 \sum_{i=1}^{n}\left(h_{i} \gamma_{i}\right)+\frac{2 c}{\tan (\varphi)}+\right. \\
\left.\left(\sigma_{1}-\sigma_{2}\right) \cos 2\left(45 \pm \frac{\theta_{\max }}{2}\right)\right] \tan (\varphi)+2 \mathrm{c}
\end{gathered}
$$

where, $\sigma_{1}, \sigma_{2}$ - values of the main stresses, MPa; $\Theta_{\max }$ - the angle determining the value of the deviation of the total stress acting on the site under consideration, from the normal to it, grad; $\mathrm{h}_{\mathrm{i}}$ - the thickness of the structural layer, $\mathrm{m} ; \gamma_{\mathrm{i}}$ - the specific gravity of the soil, $\mathrm{kN} / \mathrm{m}^{2}$

It should be noted that the left part of the inequation (9) reflects the value of the active shear stress $\tau_{\mathrm{a}}$, and the right part - the maximum permissible active shear stress $\sigma_{\max }$.

The above shown formula and prerequisites of the mechanics of granular media allow us to determine the values of the unevenness of the transmission of pressure from the wheel or die on the pavement, and also to solve the problems concerning the determination of the nature of the distribution of normal and shear stresses and their values in the underlying layers of the structure of the coating or base.

\begin{tabular}{|c|c|c|c|c|c|c|c|c|c|c|}
\hline No & $\begin{array}{c}\text { Pressure, } \\
\mathrm{MPa}\end{array}$ & $\begin{array}{l}\text { Diameter of } \\
\text { the die }, m\end{array}$ & Material & $\begin{array}{c}E \text { (elastic } \\
\text { deformation), } \\
\mathrm{MPa} \\
\end{array}$ & $\begin{array}{c}c, \\
\mathrm{MPa}\end{array}$ & $\phi$, degr & $\begin{array}{r}\gamma, \\
\mathrm{kN} / \mathrm{m}^{3}\end{array}$ & $v^{2}$ & $\mathrm{~h}_{\mathrm{i}}, \mathrm{m}$ & $\begin{array}{c}\mathrm{A}_{\mathrm{as}} \mathrm{xb}_{\mathrm{as}}, \\
\mathrm{m}\end{array}$ \\
\hline 1 & \multirow{4}{*}{0.5} & \multirow{4}{*}{0.34} & Paving stone & 1350 & - & - & - & - & 0.07 & $\begin{array}{c}0.10 \times 0.2 \\
0\end{array}$ \\
\hline 2 & & & Sand & 50 & 0 & 32 & 17 & 0.1346 & 0.05 & - \\
\hline 3 & & & Breakstone & 360 & & 42 & 19 & 0.1403 & 0.15 & - \\
\hline 4 & & & Sandy loam & 15 & 0.005 & 33 & 26 & 0.230 & - & - \\
\hline
\end{tabular}

Table 1. The source data for checking the conditions of shear resistance.

The recommendations for the design of artificial stone paving slabs [2, 3] use the assumption that in the calculations of the construction it is possible not to take into account the prefabricated coating and leveling layer. As was noted earlier this approach does not allow for the structure of the assembly (levelling) layer of sand, the geometric parameters of paving stones and features of the pressure distribution. For the comparative analysis of the results obtained and set out in the regulatory documents solutions with the results of the calculation in the proposed method $[2,3,4]$ we will use the original data presented in Table 1 as an example. The calculation is made according to the criterion of shear resistance in the loosely-coupled soil base, namely in the sandy loam.

Since the coating consists of several layers to perform the test of resistance to shear at a certain point in the structure, it is necessary to take into consideration the transmission of stresses in the subsequent layers minding the distribution of stresses in the previous ones, i.e. it is necessary to solve the problem for a multilayer structure. For this purpose we will use the formula (2), (3). The calculation scheme is shown in Figure 1.

According to the method presented earlier, we will determine the cross-section with the largest values of vertical stresses in the base soil (Figures 3 ). 


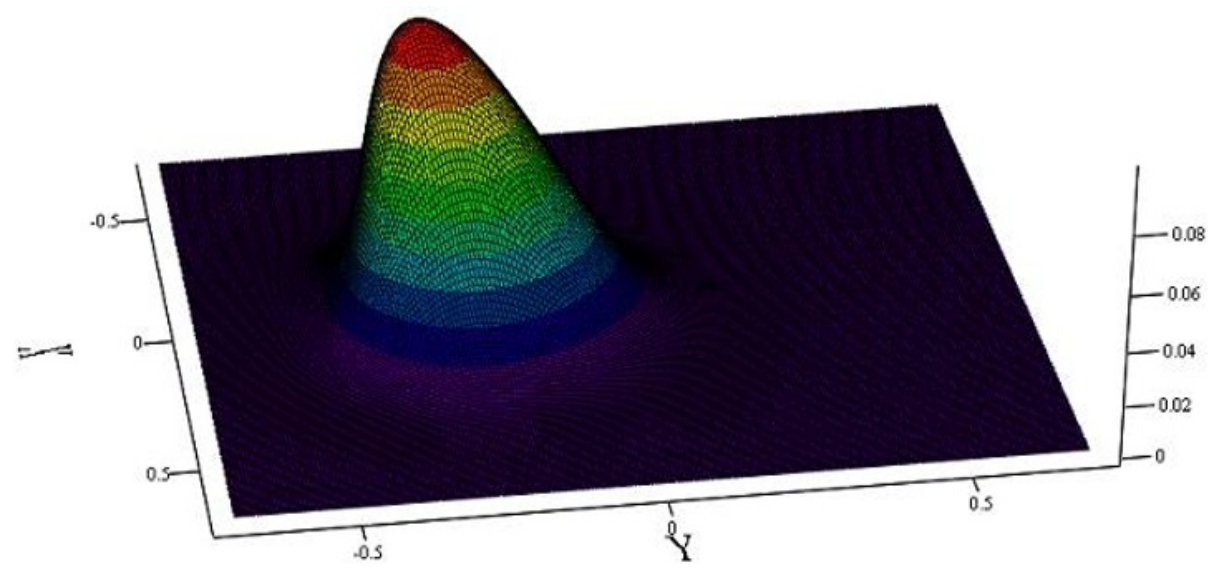

Fig. 3. The distribution of vertical stresses in the base soil at a depth of $0.27 \mathrm{~m}$ from the top of the coating.

The comparative results of the calculations performed by the current method and the proposed one are presented in Table 2.

Table 2. The results of comparative calculations.

\begin{tabular}{|c|c|c|c|c|c|}
\hline No & Soil base & Calculated value & $\begin{array}{l}\text { Unit of } \\
\text { measurement }\end{array}$ & $\begin{array}{l}\text { Calculation } \\
\text { according to [4] }\end{array}$ & $\begin{array}{l}\text { Proposed } \\
\text { method }\end{array}$ \\
\hline 1 & \multirow{7}{*}{ Sandy loam } & $\mathrm{D}_{\mathrm{p}}$ & $\mathrm{m}$ & 1.180 & - \\
\hline 2 & & $\frac{p}{\sigma_{\mathrm{z}}^{c}}$ & $\mathrm{MPa}$ & 0.0415 & 0.0925 \\
\hline 3 & & $\sigma_{\mathrm{x}}^{\mathrm{c}}$ & $\mathrm{MPa}$ & - & 0.0212 \\
\hline 4 & & $\tau_{\mathrm{xz}}^{\mathrm{c}}$ & $\mathrm{MPa}$ & - & 0.00000000433 \\
\hline 5 & & $\tau_{\lim }^{c}$ & $\mathrm{MPa}$ & 0.00956 & 0.0989 \\
\hline 6 & & $\tau_{\mathrm{a}}^{\mathrm{c}}$ & $\mathrm{MPa}$ & 0.0065 & 0.0719 \\
\hline 7 & & $\begin{array}{l}\text { The condition of } \\
\text { shear resistence }\end{array}$ & - & Being performed & Being performed \\
\hline
\end{tabular}

The following conclusions can be drawn from the results of the calculations:

- The difference in the values of vertical stresses between the normative calculation method and the results obtained by the proposed method reaches several times. Herewith, in the first case the value of the vertical pressure on the base is understated;

- In the calculation of both methods the shear stability condition is provided;

- In the calculation method [4] the critical point is on the axis of the applied load $X=0$, $\mathrm{Y}=0$, and in the compared method $-\mathrm{X}=0, \mathrm{Y}=-0.25$. As a result, when using the proposed method of calculation, the designer has a greater opportunity to correctly assign measures to ensure the criterion of shear resistance, for example, with the help of geosynthetic material with their reasonable location in the massif of the soil or by varying the thickness of the structural layers.

When using the solutions described in [4], the stresses are distributed at an angle of 45 degrees from the surface of the load application, however, this is a significant assumption since factors such as the compaction factor, the average particle size fractions, the deformation module, etc. affecting the nature of the stress distribution are not considered.

\section{Conclusion}

The paper shows that when doing the calculated checking of the provided conditions of shear resistance of the soil base coatings made from artificial stones it is necessary to consider the peculiarities of the pressure transfer from each stone individually and collectively, as it 
affects the process of determination of the most critical points in the soil. The use of the proposed method of calculation makes it possible to choose more reasonable structural measures to improve the strength and deformation characteristics of the soil, for example, with the use of geosynthetic material.

In case of further improvement of the method of checking the shear resistance of structural layers of prefabricated structures made from artificial paving stones the authors consider the following areas of research in this field as the most promising ones:

1. The study of the nature of the transmitted pressure on the base depending on the type of paving stones, the geometric parameters of the stones and the method of sealing seams;

2. The experimental determination of the dependence of the distribution coefficient on the granulometric composition of the levelling sand layer, its compaction coefficient and deformation module;

3. The development of criteria for the assigning of layers and the measures to improve the load-bearing capacity of the layers of the coating structures depending on the maximum permissible stress tensor components.

From the practical point of view for the convenience of designing such coatings it is possible to create an album of typical structures for specific road and climatic zones with ready-made solutions to strengthen the base and structural layers. In this case, the designer will need to use the proposed methodology for optimizing the thickness of the structural layers to ensure the criterion of shear resistance in the specific engineering and geological conditions.

\section{References}

1. Yu.B. Kostikov, Paving the territories of ports and logistics terminals. Design, construction, operation (St. Petersburg, 2012)

2. A.M. Simanovsky, Guide to the design, construction technology and requirements for road surfaces of artificial stones in St. Petersburg (St. Petersburg city hall, 1996)

3. Russian Standard RMD 32-18-2012.

4. Russian Standard ODN 218.046-01

5. I.I. Kandaurov, Mechanics of granular media and its application in construction: scientific publication (Leningrad, 1988)

6. N.S. Pavlova, Features of sidewalks performance with a coating from prefabricated concrete elements (Moscow, 1982)

7. A.V. Moshenzhal, Transport: science, technology, management 12 (2014)

8. A.V. Moshenzhal, Building materials, equipment, technologies of 21 st century 7, 83944 (2015)

9. N.N. Maslov, Fundamentals of engineering geology and soil mechanics: the textbook for higher schools (Higher school, Moscow, 1982)

10. A.V. Matveev, Rheological model of a granular medium and its application to the estimation of sediment of ground bases of surface foundations: Part 2 (Saint Petersburg, 2009)

11. B. Indraratha, S. Nimbalkar, Deformation Characteristics of Railway Ballast Stabilized with Geosynthetics. Testing and consulting Report (New Delhi, 2010)

12. B.M. Das, Use of Geogrid in Subgrade-Ballast System of Railroads Subjected to Cyclic Loading for Reducing Maintenance (California State University, Sacramento, 2010)

13. E. Tutumluer, H. Huang, X. Bian, Research on the behavior of geogrids in stabilization applications (Urbana, 2011) 